\title{
New properties of the time-scale fractional operators with application to dynamic equations
}

\author{
Amin Benaissa Cherif, Fatima Zohra Ladrani
}

\begin{abstract}
We introduce new properties of Riemann-Liouville fractional integral and derivative on time scales. As well as sufficient conditions for existence and uniqueness of solution to an initial value problem for a class differential equations on time scales.
\end{abstract}

\section{INTRODUCTION}

Fractional calculus was introduced and developed by Leibniz, Liouville, Riemann, Letnikov, and Grünwald [15]. This branch was applied in physics, natural and social sciences. In recent years, there has been much research activity concerning the Fractional calculus of various dynamic equations. The theory of time scales was introduced by Stefan Hilger in his PhD thesis [22] in 1988, in order to unify and generalize continuous and discrete analysis. For more detailed discussions on the time scale calculus we refer to the books (Bohner and Peterson, 2001, 2003), see [17, 18].

In 2016, Benkhettou et al. [21], introduced a concept of fractional derivative of Riemann-Liouville on time scales. Several authors have obtained important results about different subjects on time scales. See for instance M. Rchid et al [19], A. Abdeljawad et al [25], T. Gülsen et al [26].

The main purpose of this paper is to be deduced some new properties of the Riemann-Liouville fractional operator. As applications, we investigate fon existence and uniqueness of solutions some classes fractional dynamic equations.

The paper is organized as follows. In the next sections, we give some definitions and facts of time scale calculus. In Section 3, we establish some new properties of the Riemann-Liouville fractional operator. In Section 4, we investigate some IVPs for some classes fractional dynamic equations. In Section 5, we illustrate our results with examples.

2020 Mathematics Subject Classification. Primary: 26A33; Secondary: 34K37.

Key words and phrases. Time scales, fractional derivatives, dynamic equations, initial value problems.

Full paper. Received 15 April 2020, revised 12 September 2020, revised 26 January 2021, accepted 27 January 2021, available online 16 March 2021. 


\section{Preliminaries}

A time scale $\mathbb{T}$ is an arbitrary nonempty closed subset of the real numbers $\mathbb{R}$. The jump operators $\sigma, \rho: \mathbb{T} \rightarrow \mathbb{R}$ are defined by:

$$
\sigma(t):=\inf \{s \in \mathbb{T}: s>t\}, \quad \rho(t):=\inf \{s \in \mathbb{T}: s>t\} .
$$

The point $t \in \mathbb{T}$ is left-dense, left-scattered, right-dense, right-scattered if $\rho(t)=t, \rho(t)<t, \sigma(t)=t, \sigma(t)>t$, respectively. The graininess function $\mu$, for a time scale, is defined by $\mu(t)=\sigma(t)-t$. For a function $f: \mathbb{T} \rightarrow \mathbb{R}$, the function $f^{\sigma}$ denotes $f \circ \sigma$. The $\Delta$-derivative of $f: \mathbb{T} \rightarrow \mathbb{R}$ at a right dense point $t$ is defined by

$$
f^{\Delta}(t)=\lim _{s \rightarrow t} \frac{f(t)-f(s)}{t-s} .
$$

If $t$ is right scattered, then the $\Delta$-derivative is defined by

$$
f^{\Delta}(t)=\frac{f^{\sigma}(t)-f(t)}{\mu(t)} .
$$

A function $f: \mathbb{T} \rightarrow \mathbb{R}$ is said to be rd-continuous if it is continuous at each right-dense point and if there exists a finite left limit in all left-dense points. The set of rd-continuous functions $f: \mathbb{T} \rightarrow \mathbb{R}$ is denoted by $\mathcal{C}_{r d}(\mathbb{T}, \mathbb{R})$.

In what follows, with $\mathcal{C}\left([a, b]_{\mathbb{T}}, \mathbb{R}\right)$ we denote the Banach space of all continuous functions from $[a, b]_{\mathbb{T}}$ into $\mathbb{R}$, where $[a, b]_{\mathbb{T}}=[a, b] \cap \mathbb{T}$, with the norm

$$
\|x\|_{\infty}:=\sup \left\{|x(t)|: t \in[a, b]_{\mathbb{T}}\right\} .
$$

Definition 1. [17] Let $[a, b]_{\mathbb{T}}$ denote a closed bounded interval in $\mathbb{T}$. A function $F:[a, b]_{\mathbb{T}} \rightarrow \mathbb{R}$ is called a delta antiderivative function $f:[a, b]_{\mathbb{T}} \rightarrow \mathbb{R}$ provided $F$ is continuous on $[a, b]_{\mathbb{T}}$, delta differentiable on $[a, b)$, and $F^{\Delta}(t)=$ $f(t)$, for all $t \in[a, b)$. Then, we define the $\Delta$-integral of $f$ from $a$ to $b$ by

$$
\int_{a}^{b} f(t) \Delta t=F(b)-F(a) .
$$

Proposition 1. [1] Let $[a, b]_{\mathbb{T}}$ denote a closed bounded interval in $\mathbb{T}$ and $f$ is an integrable function on $[a, b]_{\mathbb{T}}$. Then

$$
\int_{a}^{b} f(t) \Delta t=\int_{[a, b]_{\mathbb{T}}} f(t) \mathrm{d} t+\sum_{t \in \mathcal{R} \cap[a, b)} \mu(t) f(t),
$$

where $\mathcal{R}=\{t \in \mathbb{T}: \sigma(t)>t\}$ is at most countable.

Definition 2 (Fractional integral on time scales). [21] Suppose $\mathbb{T}$ is a time scale, $[a, b]$ is an interval of $\mathbb{T}$, and $h$ is an integrable function on $[a, b]$. Let $0<\alpha<1$. Then the (left) fractional integral of order $\alpha$ of $h$ is defined by

$$
{ }_{a}^{\mathbb{T}} I_{t}^{\alpha} h(t)=\int_{a}^{t} \frac{(t-s)^{\alpha-1}}{\Gamma(\alpha)} h(s) \Delta s,
$$

where $\Gamma$ is the gamma function. 
Definition 3 (Riemann-Liouville fractional derivative on time scales [21]). Let $\mathbb{T}$ be a time scale, $t \in \mathbb{T}, 0<\alpha<1$, and $h: \mathbb{T} \rightarrow \mathbb{R}$. The (left) Riemann-Liouville fractional derivative of order $\alpha$ of $h$ is defined by

$$
{ }_{a}^{\mathbb{T}} D_{t}^{\alpha} h(t)=\left(\int_{a}^{t} \frac{(t-s)^{-\alpha}}{\Gamma(1-\alpha)} h(s) \Delta s\right)^{\Delta}=\left({ }_{a}^{\mathbb{T}} I_{t}^{1-\alpha} h(t)\right)^{\Delta} .
$$

Proposition 2. If $\mathbb{T}=\mathbb{R}$, the Riemann-Liouville fractional integral satisfies

$$
{ }_{a}^{\mathbb{T}} I_{t}^{\beta} \circ{ }_{a}^{\mathbb{T}} I_{t}^{\alpha}={ }_{a}^{\mathbb{T}} I_{t}^{\beta+\alpha}, \quad \text { for } \alpha>0 \text { and } \beta>0 .
$$

\section{Main Results}

In this section, we present some of the new properties of the time-scale fractional operators.

The following counter example, to prove that the equality (1) is not always satisfied on time scales.

Example 1 (Counter example). We take $\mathbb{T}=\mathbb{N}, a=1$ and $h: \mathbb{T} \rightarrow \mathbb{R}$, $h(t)=1$. Let $\alpha>0$ and $b>0$, then by Definition 2 , we have

$$
\begin{aligned}
{ }_{1}^{\mathbb{N}} I_{t}^{\alpha} h(t) & =\frac{1}{\Gamma(\alpha)} \int_{1}^{t}(t-s)^{\alpha-1} \Delta s=\frac{1}{\Gamma(\alpha)} \sum_{s=1}^{s=t-1}(t-s)^{\alpha-1} \\
& =\frac{1}{\Gamma(\alpha)}\left[(t-1)^{\alpha-1}+(t-2)^{\alpha-1}+\cdots+1^{\alpha-1}\right] \\
& =\frac{1}{\Gamma(\alpha)} \sum_{s=1}^{s=t-1} s^{\alpha-1}:=\varphi(t) .
\end{aligned}
$$

By, the last equality, deduce

$$
{ }_{1}^{\mathbb{N}} I_{t}^{\alpha+\beta} h(t)=\frac{1}{\Gamma(\alpha+\beta)} \sum_{s=1}^{s=t-1} s^{\alpha+\beta-1} .
$$

On the other hand, we have

$$
\begin{aligned}
\left({ }_{1}^{\mathbb{T}} I_{t}^{\beta} \circ \frac{\mathbb{T}}{1} I_{t}^{\alpha}\right) h(t) & ={ }_{1}^{\mathbb{N}} I_{t}^{\beta} \varphi(t)=\frac{1}{\Gamma(\beta)} \int_{1}^{t}(t-s)^{\beta-1} \varphi(s) \Delta s \\
& =\frac{1}{\Gamma(\beta)} \sum_{s=1}^{s=t-1}(t-s)^{\beta-1} \varphi(s) .
\end{aligned}
$$

Thus,

$$
\left({ }_{1}^{\mathbb{T}} I_{t}^{\beta} \circ_{1}^{\mathbb{T}} I_{t}^{\alpha}\right) h(2)=\frac{1}{\Gamma(\alpha) \Gamma(\beta)} \quad \text { and } \quad{ }_{1}^{\mathbb{N}} I_{t}^{\alpha+\beta} h(2)=\frac{1}{\Gamma(\alpha+\beta)}
$$

and

$$
\left({ }_{1}^{\mathbb{T}} I_{t}^{\beta} \circ_{1}^{\mathbb{T}} I_{t}^{\alpha}\right) h(2) \neq{ }_{1}^{\mathbb{T}} I_{t}^{\alpha+\beta} h(2)
$$


Remark 1. By counter example 1, we conclude that ${ }_{a}^{\mathbb{T}} I_{t}^{\beta} \circ{ }_{a}^{\mathbb{T}} I_{t}^{\alpha}={ }_{a}^{\mathbb{T}} I_{t}^{\beta+\alpha}$, for $\alpha>0, \beta>0$ are not always correct on the time scales, which are proposition 16 in [21]. If you suggest a counterexample to ${ }_{a}^{\mathbb{T}} D_{t}^{\alpha} \circ{ }_{a}^{\mathbb{T}} I_{t}^{\alpha}=I d$ and ${ }_{a}^{\mathbb{T}} I_{t}^{\alpha} \circ{ }_{a}^{\mathbb{T}} D_{t}^{\alpha}=I d, \alpha>0$, you leave to provide exact calculations in Example 1. Example 1 is a for ${ }_{a}^{\mathbb{T}} I_{t}^{\beta} \circ{ }_{a}^{\mathbb{T}} I_{t}^{\alpha} \neq{ }_{a}^{\mathbb{T}} I_{t}^{\beta+\alpha}$ only.

Before starting to introduce the properties of the time scale fractional operators, we present a new generalization for the Beta function on time scales.

Definition 4 (Beta function on time scales). We will define the function $B_{a, b}^{\mathbb{T}}(\alpha, \beta)$ as follows

$$
B_{a, b}^{\mathbb{T}}(\alpha, \beta)=\int_{a}^{b}(s-a)^{\beta-1}(b-s)^{\alpha-1} \Delta s, \quad \text { for } \alpha>0, \beta>0 .
$$

Remark 2. If $\mathbb{T}=\mathbb{R}, a=0$ and $b=1$, then Definition 4 takes the form

$$
B_{0,1}^{\mathbb{R}}(\alpha, \beta)=B(\alpha, \beta), \quad \text { for } \alpha>0 \quad \text { and } \beta>0,
$$

where $B$ is the classical beta function.

Proposition 3. The function $B_{a, b}^{\mathbb{T}}(\alpha, \beta)$ satisfies the following inequality

$$
B_{a, b}^{\mathbb{T}}(\alpha, \beta) \geq B(\alpha, \beta)(b-a)^{\alpha+\beta-1}, \quad \text { for } \alpha>0 \quad \text { and } \beta>0 .
$$

Proof. By Proposition 1, we have

$$
B_{a, b}^{\mathbb{T}}(\alpha, \beta) \geq \int_{a}^{b}(s-a)^{\beta-1}(b-s)^{\alpha-1} d s=B_{a, b}^{\mathbb{R}}(\alpha, \beta) .
$$

By setting $s=a+r(b-a), r \in[0,1]$, we obtain that

$$
\begin{aligned}
B_{a, b}^{\mathbb{T}}(\alpha, \beta) & \geq(b-a)^{\alpha+\beta-1} \int_{a}^{b} r^{\beta-1}(1-r)^{\alpha-1} \mathrm{~d} s \\
& =(b-a)^{\alpha+\beta-1} B_{0,1}^{\mathbb{R}}(\alpha, \beta) \\
& =B(\alpha, \beta)(b-a)^{\alpha+\beta-1} .
\end{aligned}
$$

The proof is complete.

Example 2. If $\mathbb{T}=\mathbb{N}$, let $a, b \in \mathbb{N}$, by Definition 4 , we have

$$
B_{a, b}^{\mathbb{N}}(\alpha, \beta)=\sum_{s=a}^{s=b-1}(s-a)^{\beta-1}(b-s)^{\alpha-1}, \quad \text { for } \alpha>1, \beta>1 .
$$

Definition 5. Let $\lambda \in \mathbb{R}$ and $a \in \mathbb{R}$ we define the time scales $\lambda \mathbb{T}$ and $\mathbb{T}+a$ by:

$$
\lambda \mathbb{T}:=\{\lambda t: t \in \mathbb{T}\}, \quad \mathbb{T}+a:=\{t+a: t \in \mathbb{T}\} .
$$

Definition 6. Let $\lambda \in \mathbb{R}, a \in \mathbb{R}$ and let be the function $v: \mathbb{T} \rightarrow \lambda(\mathbb{T}+a)$ defined by

$$
v_{\lambda, a}(s)=\lambda(s+a), \quad \text { for all } s \in \mathbb{T} .
$$


Remark 3. Let $\lambda \in \mathbb{R}$ and $a \in \mathbb{R}$, such as $\lambda \neq 0$, then function $v_{\lambda, a}$ is bijective and the inverse function $v_{\lambda, a}^{-1}$ given by

$$
v_{\lambda, a}^{-1}(s)=\frac{s}{\lambda}-a=v_{\frac{1}{\lambda},-a \lambda}(s), \quad \text { for all } s \in \lambda(\mathbb{T}+a) .
$$

Notation 1. We define the time scales $\mathbb{T}_{a, b}$ by:

$$
\mathbb{T}_{a, b}:=\frac{1}{b-a}(\mathbb{T}-a) .
$$

Proposition 4. The Beta function on time scales satisfies the following useful property:

$$
B_{a, b}^{\mathbb{T}}(\alpha, \beta)=(b-a)^{\beta+\alpha-1} \beta_{0,1}^{\mathbb{T}_{a, b}}(\beta, \alpha), \quad \text { for } \alpha>0 \quad \text { and } \beta>0,
$$

where

$$
\beta_{0,1}^{\mathbb{T}_{a, b}}(\beta, \alpha)=\int_{0}^{1} r^{\beta-1}(1-r)^{\alpha-1} \Delta_{\mathbb{T}_{a, b}} r
$$

Proof. The function $u_{a, b}=v_{\frac{1}{b-a},-a}$ is bijective and the inverse function $u_{a, b}^{-1}$ given by

$$
u_{a, b}^{-1}(r)=a+r(b-a), \quad \text { for all } r \in \mathbb{T}_{a, b} .
$$

By the chain rule [17], we see that

$$
\begin{aligned}
B_{a, b}^{\mathbb{T}}(\alpha, \beta) & =(b-a) \int_{u_{a, b}(a)}^{u_{a, b}(b)}\left[(s-a)^{\beta-1}(b-s)^{\alpha-1}\right] \circ u_{a, b}^{-1}(r) \Delta_{\mathbb{T}_{a, b}} r \\
& =(b-a)^{\beta+\alpha-1} \int_{0}^{1} r^{\beta-1}(1-r)^{\alpha-1} \Delta_{\mathbb{T}_{a, b}} r \\
& =(b-a)^{\beta+\alpha-1} \beta_{0,1}^{\mathbb{T}_{a, b}}(\beta, \alpha) .
\end{aligned}
$$

The proof is complete.

Proposition 5. For any function $h$ integrable on $[a, b]_{\mathbb{T}}$, the RiemannLiouville $\Delta$-fractional integral satisfies

$$
\left({ }_{a}^{\mathbb{T}} I_{t}^{\beta} \circ{ }_{a}^{\mathbb{T}} I_{t}^{\alpha}\right)(h(t))=\frac{1}{\Gamma(\alpha+\beta)} \int_{a}^{t} h(u)(t-u)^{\beta+\alpha-1} \frac{\beta_{0,1}^{\mathbb{T}_{u, t}}(\beta, \alpha)}{B(\alpha, \beta)} \Delta u,
$$

for $\alpha>0$ and $\beta>0$, where $\beta_{0,1}^{\mathbb{T}_{u, t}}(\beta, \alpha)$ is defined as in Proposition 4 .

Proof. By Definition 4, we get

$$
\begin{aligned}
\left({ }_{a}^{\mathbb{T}} I_{t}^{\alpha} \circ_{a}^{\mathbb{T}} I_{t}^{\beta}\right)(h(t)) & ={ }_{a}^{\mathbb{T}} I_{t}^{\alpha}\left({ }_{a}^{\mathbb{T}} I_{t}^{\beta} h(t)\right) \\
& =\frac{1}{\Gamma(\alpha)} \int_{a}^{t}(t-s)^{\alpha-1}\left({ }_{a}^{\mathbb{T}} I_{t}^{\beta} h(s)\right) \Delta s \\
& =\frac{1}{\Gamma(\alpha) \Gamma(\beta)} \int_{a}^{t}(t-s)^{\alpha-1}\left(\int_{a}^{s}(s-u)^{\beta-1} h(u) \Delta u\right) \Delta s .
\end{aligned}
$$


From Fubini's theorem, we interchange the order of integration to obtain

$$
\begin{aligned}
\left({ }_{a}^{\mathbb{T}} I_{t}^{\alpha} \circ_{a}^{\mathbb{T}} I_{t}^{\beta}\right)(h(t)) & =\frac{1}{\Gamma(\alpha) \Gamma(\beta)} \int_{a}^{t} h(u)\left(\int_{u}^{t}(s-u)^{\beta-1}(t-s)^{\alpha-1} \Delta s\right) \Delta u \\
& =\frac{1}{\Gamma(\alpha) \Gamma(\beta)} \int_{a}^{t} h(u) B_{u, t}^{\mathbb{T}}(\alpha, \beta) \Delta u .
\end{aligned}
$$

By Proposition 4, we obtain that

$$
\begin{aligned}
\left({ }_{a}^{\mathbb{T}} I_{t}^{\alpha} \circ \underset{a}{\mathbb{T}} I_{t}^{\beta}\right)(h(t)) & =\frac{1}{\Gamma(\alpha) \Gamma(\beta)} \int_{a}^{t} h(u)(t-u)^{\beta+\alpha-1} \beta_{0,1}^{\mathbb{T}_{u, t}}(\beta, \alpha) \Delta u \\
& =\frac{1}{\Gamma(\alpha+\beta)} \int_{a}^{t} h(u)(t-u)^{\beta+\alpha-1} \frac{\beta_{0,1}^{\mathbb{T}_{u, t}}(\beta, \alpha)}{B(\alpha, \beta)} \Delta u .
\end{aligned}
$$

The proof is complete.

Remark 4. If $\mathbb{T}=\mathbb{R}$, we have $\beta_{0,1}^{\mathbb{T}_{u, t}}(\beta, \alpha)=B(\alpha, \beta)$, then Proposition 5 gives the classic result.

Corollary 1. For any function $h$ integrable and positive on $[a, b]_{\mathbb{T}}$, the Riemann-Liouville $\Delta$-fractional integral satisfies

$$
\left({ }_{a}^{\mathbb{T}} I_{t}^{\beta} \circ{ }_{a}^{\mathbb{T}} I_{t}^{\alpha}\right)(h(t)) \geq_{a}^{\mathbb{T}} I_{t}^{\beta+\alpha}(h(t)),
$$

for $\alpha>0$ and $\beta>0$.

Proof. Form Proposition 5 and Proposition 3, we have

$$
\begin{aligned}
\left({ }_{a}^{\mathbb{T}} I_{t}^{\beta} \circ_{a}^{\mathbb{T}} I_{t}^{\alpha}\right)(h(t)) & =\frac{1}{\Gamma(\alpha+\beta)} \int_{a}^{t} h(u)(t-u)^{\beta+\alpha-1} \frac{\beta_{0,1}^{\mathbb{T}_{u, t}}(\beta, \alpha)}{B(\alpha, \beta)} \Delta u \\
& \geq \frac{1}{\Gamma(\alpha+\beta)} \int_{a}^{t} h(u)(t-u)^{\beta+\alpha-1} \Delta u \\
& ={ }_{a}^{\mathbb{T}} I_{t}^{\beta+\alpha}(h(t)) .
\end{aligned}
$$

The proof is complete.

Proposition 6. Let $h:[a, b]_{\mathbb{T}} \rightarrow \mathbb{R}$ be a function $\Delta$ is differentiable, such that $h^{\Delta}$ integrable on $[a, b]_{\mathbb{T}}$. Then

$$
{ }_{a}^{\mathbb{T}} I_{t}^{\alpha} h^{\Delta}(t)=\frac{1}{\Gamma(\alpha-1)} \int_{a}^{t}(t-s)^{\alpha-2} \eta(t, s) h^{\sigma}(s) \Delta s-\frac{h(a)}{\Gamma(\alpha)}(t-a)^{\alpha-1},
$$

for all $\alpha>1$, with $\eta(t, s)=\int_{0}^{1}\left[1-h \frac{\mu(s)}{t-s}\right]^{\alpha-2} d h$.

Proof. By Definition 2, we have

$$
\begin{aligned}
{ }_{a}^{\mathbb{T}} I_{t}^{\alpha} h^{\Delta}(t) & =\int_{a}^{t} \frac{(t-s)^{\alpha-1}}{\Gamma(\alpha)} h^{\Delta}(s) \Delta s \\
& =\int_{a}^{t} \frac{(t-s)^{\alpha-1}}{\Gamma(\alpha)}[h(s)-h(t)]^{\Delta} \Delta s
\end{aligned}
$$




$$
\begin{aligned}
= & \frac{1}{\Gamma(\alpha)}\left[(t-s)^{\alpha-1}[h(s)-h(t)]\right]_{a}^{t} \\
& -\frac{1}{\Gamma(\alpha)} \int_{a}^{t}\left[(t-s)^{\alpha-1}\right]^{\Delta_{s}}\left[h^{\sigma}(s)-h(t)\right] \Delta s \\
= & -\frac{1}{\Gamma(\alpha)} \int_{a}^{t}\left[(t-s)^{\alpha-1}\right]^{\Delta_{s}}\left[h^{\sigma}(s)-h(t)\right] \Delta s \\
& -\frac{1}{\Gamma(\alpha)}(t-a)^{\alpha-1}[h(a)-h(t)] .
\end{aligned}
$$

By Pötzsche's chain rule, we have

$$
\begin{aligned}
-\frac{1}{\Gamma(\alpha)}\left[(t-s)^{\alpha-1}\right]^{\Delta_{s}} & =\frac{\alpha-1}{\Gamma(\alpha)} \int_{0}^{1}[(1-h)(t-s)+h(t-\sigma(s))]^{\alpha-2} \mathrm{~d} h \\
& =\frac{(t-s)^{\alpha-2}}{\Gamma(\alpha-1)} \int_{0}^{1}\left[1-h+h \frac{t-\sigma(s)}{t-s}\right]^{\alpha-2} \mathrm{~d} h \\
& =\frac{(t-s)^{\alpha-2}}{\Gamma(\alpha-1)} \int_{0}^{1}\left[1-h \frac{\mu(s)}{t-s}\right]^{\alpha-2} \mathrm{~d} h \\
& =\frac{(t-s)^{\alpha-2}}{\Gamma(\alpha-1)} \eta(t, s) .
\end{aligned}
$$

Then,

$$
\begin{aligned}
{ }_{a}^{\mathbb{T}} I_{t}^{\alpha} h^{\Delta}(t)= & \frac{1}{\Gamma(\alpha-1)} \int_{a}^{t}(t-s)^{\alpha-2} \eta(t, s) h^{\sigma}(s) \Delta s \\
& +\frac{h(t)}{\Gamma(\alpha)} \int_{a}^{t}\left[(t-s)^{\alpha-1}\right]^{\Delta_{s}} \Delta s-\frac{1}{\Gamma(\alpha)}(t-a)^{\alpha-1}[h(a)-h(t)] \\
= & \frac{1}{\Gamma(\alpha-1)} \int_{a}^{t}(t-s)^{\alpha-2} \eta(t, s) h^{\sigma}(s) \Delta s-\frac{h(t)}{\Gamma(\alpha)}(t-a)^{\alpha-1} \\
& -\frac{1}{\Gamma(\alpha)}(t-a)^{\alpha-1}[h(a)-h(t)] \\
= & \frac{1}{\Gamma(\alpha-1)} \int_{a}^{t}(t-s)^{\alpha-2} \eta(t, s) h^{\sigma}(s) \Delta s-\frac{h(a)}{\Gamma(\alpha)}(t-a)^{\alpha-1} .
\end{aligned}
$$

The proof is complete.

Corollary 2. Let $h:[a, b]_{\mathbb{T}} \rightarrow \mathbb{R}$ be a function $\Delta$ is differentiable, such that $h^{\Delta}$ integrable on $[a, b]_{\mathbb{T}}$, then

$$
\left|{ }_{a}^{\mathbb{T}} I_{t}^{\alpha} h^{\Delta}(t)\right| \leq\left|{ }_{a}^{\mathbb{T}} I_{t}^{\alpha-1} h(t)\right|+\frac{|h(a)|}{\Gamma(\alpha)}(t-a)^{\alpha-1},
$$

for all $\alpha>1$. 
Proof. Form Proposition 6, we have

$$
\begin{aligned}
\left|{ }_{a}^{\mathbb{T}} I_{t}^{\alpha} h^{\Delta}(t)\right| & \leq \frac{1}{\Gamma(\alpha-1)} \int_{a}^{t}(t-s)^{\alpha-2}\left|h^{\sigma}(s)\right| \Delta s+\frac{|h(a)|}{\Gamma(\alpha)}(t-a)^{\alpha-1} \\
& =\left|{ }_{a}^{\mathbb{T}} I_{t}^{\alpha-1} h(t)\right|+\frac{|h(a)|}{\Gamma(\alpha)}(t-a)^{\alpha-1} .
\end{aligned}
$$

The proof is complete.

We consider the following initial value problem:

$$
\left\{\begin{array} { l } 
{ \lambda x ^ { \Delta } ( t ) + { } _ { a } ^ { \mathbb { T } } D _ { t } ^ { \alpha } ( p ( t ) x ( t ) ) = f ( t , x ( t ) ) , } \\
{ \lambda x ^ { \sigma } ( a ) + ( { } _ { a } ^ { \mathbb { T } } I _ { t } ^ { 1 - \alpha } ) ^ { \sigma } ( p ( a ) x ( a ) ) = 0 , }
\end{array} \text { for } \left\{\begin{array}{l}
t \in[\sigma(a), b]_{\mathbb{T}}, \\
0<\alpha<1,
\end{array}\right.\right.
$$

where ${ }_{a}^{\mathbb{T}} D_{t}^{\alpha}$ is the Riemann-Liouville fractional derivative operator of order $\alpha$ defined on $\mathbb{T}$. The problem (2) will be studied under the following assumptions $f \in \mathcal{C}\left([\sigma(a), b]_{\mathbb{T}} \times \mathbb{R}, \mathbb{R}\right), p \in \mathcal{C}\left([\sigma(a), b]_{\mathbb{T}}, \mathbb{R}\right)$, and $\lambda \in \mathbb{R}-\{0\}$. Our main results give necessary and sufficient conditions for the existence and uniqueness of solution to the problem (2).

Lemma 1. Let $\alpha \in(0,1)$ and $f:[\sigma(a), b]_{\mathbb{T}} \times \mathbb{R} \rightarrow \mathbb{R}$. Function $x \in$ $\mathcal{C}\left([\sigma(a), b]_{\mathbb{T}}, \mathbb{R}\right)$ is a solution of the problem $(2)$ if and only if it is a solution of the following integral equation:

$$
x(t)=\frac{1}{\lambda} \int_{\sigma(a)}^{t} f(s, x(s)) \Delta s-\frac{1}{\lambda \Gamma(1-\alpha)} \int_{a}^{t}(t-s)^{-\alpha} p(s) x(s) \Delta s .
$$

Proof. By Definition 2, we have

$$
\begin{aligned}
\lambda x^{\Delta}(t) & =f(t, x(t))-\frac{1}{\Gamma(1-\alpha)}\left(\int_{a}^{t}(t-s)^{-\alpha} p(s) x(s) \Delta s\right)^{\Delta} \\
& =f(t, x(t))-\left({ }_{a}^{\mathbb{T}} I_{t}^{1-\alpha} p(t) x(t)\right)^{\Delta} \\
& =f(t, x(t))-{ }_{a}^{\mathbb{T}} D_{t}^{\alpha}(p(t) x(t)) .
\end{aligned}
$$

The proof is complete.

Our first main result is based on the Banach fixed point theorem [2].

Theorem 1. Let $\alpha \in(0,1)$ and $f \in \mathcal{C}\left([\sigma(a), b]_{\mathbb{T}} \times \mathbb{R}, \mathbb{R}\right)$, there exists a positive and continuous function $r:[\sigma(a), b]_{\mathbb{T}} \rightarrow \mathbb{R}$, such that

$$
|f(t, x)-f(t, y)| \leq r(t)|x-y| \text {. }
$$

for all $x, y \in \mathbb{R}$ and $t \in[\sigma(a), b]_{\mathbb{T}}$.

If

$$
\left({ }_{a}^{\mathbb{T}} I_{t}^{1}|p(t)|+{ }_{a}^{\mathbb{T}} I_{t}^{1-\alpha}|p(t)|\right)<|\lambda|, \quad \text { for all } t \in[\sigma(a), b]_{\mathbb{T}},
$$

then the problem (2) has a unique solution on $[\sigma(a), b]_{\mathbb{T}}$. 
Proof. We transform the problem (2) into a fixed point problem. Consider the operator $L: \mathcal{C}\left([\sigma(a), b]_{\mathbb{T}}, \mathbb{R}\right) \rightarrow \mathcal{C}\left([\sigma(a), b]_{\mathbb{T}}, \mathbb{R}\right)$ defined by

$$
L x(t)=\frac{1}{\lambda} \int_{\sigma(a)}^{t}\left(f(s, x(s))-\frac{1}{\Gamma(1-\alpha)}(t-s)^{-\alpha} p(s) x(s)\right) \Delta s .
$$

We need to prove that $L$ has a fixed point, which is a unique solution of (2) on $[\sigma(a), b]_{\mathbb{T}}$. For that, we show that $F$ is a contraction. Let $x, y \in$ $\mathcal{C}\left([\sigma(a), b]_{\mathbb{T}}, \mathbb{R}\right)$. For $t \in[\sigma(a), b]_{\mathbb{T}}$, we have

$$
\begin{aligned}
|L x(t)-L y(t)| \leq & \frac{1}{|\lambda|} \int_{\sigma(a)}^{t}|f(s, x(s))-f(s, y(s))| \Delta s \\
& +\frac{1}{\Gamma(1-\alpha)} \int_{a}^{t}(t-s)^{-\alpha}|p(s)||x(s)-y(s)| \Delta s \\
\leq & \frac{1}{|\lambda|}\left({ }_{a}^{\mathbb{T}} I_{t}^{1}|p(t)|+{ }_{a}^{\mathbb{T}} I_{t}^{1-\alpha}|p(t)|\right)\|x-y\| .
\end{aligned}
$$

By (4), $L$ is a contraction and thus, by the contraction mapping theorem, we deduce that $L$ has a unique fixed point. This fixed point is the unique solution of (2).

Now, we give our second main result guarantees the existence of at least one solution of the problem (2). This result is based on the Schauder's fixed point theorem [2].

Theorem 2. Let $\alpha \in(0,1)$ and $f \in \mathcal{C}\left([\sigma(a), b]_{\mathbb{T}} \times \mathbb{R}, \mathbb{R}\right)$, there are two functions $r \in \mathcal{C}\left([\sigma(a), b]_{\mathbb{T}},[0, \infty)\right)$ and $\varphi \in \mathcal{C}(\mathbb{R},[0, \infty))$, such that

$$
|f(t, x)| \leq r(t) \varphi(x), \quad \text { for all } y \in \mathbb{R} \quad \text { and } \quad t \in[\sigma(a), b]_{\mathbb{T}} .
$$

Then the problem (2) has a solution on $[\sigma(a), b]_{\mathbb{T}}$.

Proof. We use Schauder's fixed point theorem to prove that $L$ defined by (5) has a fixed point. The proof is given in several steps.

Step 1: $L$ is continuous. Let $x_{n}$ be a sequence such that $x_{n} \rightarrow x$ in $\mathcal{C}\left([\sigma(a), b]_{\mathbb{T}}, \mathbb{R}\right)$. Then, for each $t \in[\sigma(a), b]_{\mathbb{T}}$,

$$
\begin{aligned}
\left|L x_{n}(t)-L x(t)\right| \leq & \frac{1}{|\lambda|} \int_{\sigma(a)}^{t}\left|f\left(s, x_{n}(s)\right)-f(s, x(s))\right| \Delta s \\
& +\frac{1}{\Gamma(1-\alpha)} \int_{a}^{t}(t-s)^{-\alpha}|p(s)|\left|x_{n}(s)-x(s)\right| \Delta s \\
\leq & \frac{b-\sigma(a)}{|\lambda|} \sup _{t \in[\sigma(a), b]_{\mathbb{T}}}\left|f\left(s, x_{n}(s)\right)-f(s, x(s))\right| \\
& +\sup _{t \in[a, b]_{\mathbb{T}}}\left({ }_{a}^{\mathbb{T}} I_{t}^{1-\alpha}|p(t)|\right)\left\|x_{n}-x\right\|
\end{aligned}
$$




$$
\begin{aligned}
= & \frac{b-\sigma(a)}{|\lambda|}\left\|f\left(., x_{n}(.)\right)-f(s, x(.))\right\| \\
& +\left\|{ }_{a}^{\mathbb{T}} I_{t}^{1-\alpha}|p|\right\|\left\|x_{n}-x\right\| .
\end{aligned}
$$

Since $f$ is a continuous function, we have $L x_{n} \rightarrow L x$ in $\mathcal{C}\left([\sigma(a), b]_{\mathbb{T}}, \mathbb{R}\right)$.

Step 2: The map $L$ maps bounded sets into bounded sets in $\mathcal{C}\left([\sigma(a), b]_{\mathbb{T}}, \mathbb{R}\right)$. Indeed, it is enough to show that for any $\varepsilon$ there exists a positive constant $\delta$ such that, for each $x \in B(0, \varepsilon)$, we have $L x \in B(0, \delta)$. By hypothesis, for each $t \in[\sigma(a), b]_{\mathbb{T}}$, we get

$$
\begin{aligned}
|L x(t)| \leq & \frac{1}{|\lambda|} \int_{\sigma(a)}^{t} r(s) \varphi(x(s)) \Delta s \\
& +\frac{1}{|\lambda| \Gamma(1-\alpha)} \int_{a}^{t}(t-s)^{-\alpha}|p(s)||x(s)| \Delta s \\
\leq & \frac{1}{|\lambda|} \max _{x \in[-\varepsilon, \varepsilon]} \varphi(x) \int_{\sigma(a)}^{b} r(s) \Delta s+\frac{\varepsilon}{|\lambda|}\left\|{ }_{a}^{\mathbb{T}} I_{t}^{1-\alpha}|p|\right\|=\delta .
\end{aligned}
$$

Step 3: The map $L$ maps bounded sets into equicontinuous sets of $\mathcal{C}\left([\sigma(a), b]_{\mathbb{T}}\right.$, $\mathbb{R})$. Let $t_{1}, t_{2} \in[\sigma(a), b]_{\mathbb{T}}, t_{1}<t_{2}$ and $B(0, \varepsilon)$ be a bounded set of $\mathcal{C}\left([\sigma(a), b]_{\mathbb{T}}, \mathbb{R}\right)$. For all $x \in B(0, \varepsilon)$, we get

$$
\begin{aligned}
\left|L x\left(t_{2}\right)-L x\left(t_{1}\right)\right| \leq & \frac{1}{|\lambda|} \int_{t_{1}}^{t_{2}} r(s) \varphi(x(s)) \Delta s \\
& +\frac{1}{|\lambda| \Gamma(1-\alpha)} \int_{a}^{t_{2}}\left(t_{2}-s\right)^{-\alpha}|p(s)||x(s)| \Delta s \\
& -\frac{1}{|\lambda| \Gamma(1-\alpha)} \int_{a}^{t_{1}}\left(t_{1}-s\right)^{-\alpha}|p(s)||x(s)| \Delta s \\
\leq & \frac{1}{|\lambda|} \max _{x \in[-\varepsilon, \varepsilon]} \varphi(x) \int_{t_{1}}^{t_{2}} r(s) \Delta s \\
& +\frac{\varepsilon\|p\|}{|\lambda| \Gamma(1-\alpha)} \int_{t_{1}}^{t_{2}}\left(t_{2}-s\right)^{-\alpha} \Delta s \\
+ & \frac{\varepsilon\|p\|}{|\lambda| \Gamma(1-\alpha)} \int_{a}^{t_{1}}\left|\left(t_{2}-s\right)^{-\alpha}-\left(t_{1}-s\right)^{-\alpha}\right| \Delta s .
\end{aligned}
$$

On the other hand, by Proposition 1, we get

$$
\begin{aligned}
\int_{t_{1}}^{t_{2}}\left(t_{2}-s\right)^{-\alpha} \Delta s & \leq \int_{t_{1}}^{t_{2}}\left(t_{2}-s\right)^{-\alpha} \mathrm{d} s+\sum_{s \in\left[t_{1}, t_{2}\right) \cap \mathcal{R}} \mu(s)\left(t_{2}-s\right)^{-\alpha} \\
& \leq \frac{1}{1-\alpha}\left(t_{2}-t_{1}\right)^{1-\alpha}+\sum_{s \in\left[t_{1}, t_{2}\right) \cap \mathcal{R}}\left(t_{2}-s\right)^{1-\alpha}
\end{aligned}
$$




$$
\leq \frac{1}{1-\alpha}\left(t_{2}-t_{1}\right)^{1-\alpha}+\left(t_{2}-t_{1}\right)^{2-\alpha}
$$

Similarly, we get

$$
\begin{aligned}
& \int_{a}^{t_{1}}\left|\left(t_{2}-s\right)^{-\alpha}-\left(t_{1}-s\right)^{-\alpha}\right| \Delta s \\
\leq & \frac{1}{1-\alpha}\left[\left(t_{2}-t_{1}\right)^{1-\alpha}+\left(t_{2}-a\right)^{1-\alpha}\right. \\
& \left.-\left(t_{1}-a\right)^{1-\alpha}\right]+\sum_{s \in\left[a, t_{1}\right) \cap \mathcal{R}}\left(t_{1}-s\right)^{1-\alpha} \\
& -\sum_{s \in\left[a, t_{1}\right) \cap \mathcal{R}}\left(t_{1}-s\right)\left(t_{2}-s\right)^{-\alpha} .
\end{aligned}
$$

As $t_{1} \rightarrow t_{2}$, the right-hand side of the above inequality tends to zero. As a consequence of Steps 1 to 3, together with the Arzela-Ascoli theorem, we conclude that $L: \mathcal{C}\left([\sigma(a), b]_{\mathbb{T}}, \mathbb{R}\right) \rightarrow \mathcal{C}\left([\sigma(a), b]_{\mathbb{T}}, \mathbb{R}\right)$ is completely continuous. As a consequence of Schauder's fixed point theorem, we conclude that $L$ has a fixed point, which is solution of the problem (2).

\section{Conclusion}

If we take $\lambda=0$ in the problem (2), we get

$$
\left\{\begin{array}{l}
{ }_{a}^{\mathbb{T}} D_{t}^{\alpha}(p(t) x(t))=f(t, x(t)), \text { for } t \in[\sigma(a), b]_{\mathbb{T}}, \alpha \in(0,1), \\
\left({ }_{a}^{\mathbb{T}} I_{t}^{1-\alpha}\right)^{\sigma}(p(a) x(a))=0 .
\end{array}\right.
$$

We consider the following integral equation

$$
x(t)=\frac{1}{\Gamma(\alpha)} \int_{a}^{t}(t-s)^{\alpha-1} p(s) f(s, x(s)) \Delta s, \text { for } t \in[\sigma(a), b]_{\mathbb{T}} .
$$

Since ${ }_{a}^{\mathbb{T}} D_{t}^{\alpha} \circ_{a}^{\mathbb{T}} I_{t}^{\alpha}=I d$, and ${ }_{a}^{\mathbb{T}} I_{t}^{\alpha} \circ_{a}^{\mathbb{T}} D_{t}^{\alpha}=I d$, for $\alpha>0$ are not always correct defined on the time scales. Then, if $x$ is a solution to the problem (6), it has no permanent relationship the solution of integral equation (7).

\section{ExAmple}

Remark 5. Let $f:[a, b]_{\mathbb{T}} \rightarrow \mathbb{R}$ and $a$ is right-scattered. By Definition 2, we have

$$
\left({ }_{a}^{\mathbb{T}} I_{t}^{\alpha} h\right)^{\sigma}(a)=\int_{a}^{\sigma(a)} \frac{(\sigma(a)-s)^{\alpha-1}}{\Gamma(\alpha)} h(s) \Delta s=\frac{(\mu(a))^{\alpha} h(a)}{\Gamma(\alpha)} .
$$

Example 3. Let $\mathbb{T}=h \mathbb{Z}, a=h$ and $b=m h$, where $h>1$ and $m \in \mathbb{N}$. Then $\sigma(t)=t+h$ and $\mu(t)=0$.

We consider the following initial value problem:

$$
\left\{\begin{array}{l}
\lambda x^{\Delta}(t)+{ }_{h}^{h \mathbb{Z}} D_{t}^{\frac{1}{2}} x(t)=t^{2} x(t), \quad \text { for } t \in[h, m h]_{h \mathbb{Z}}, \\
\lambda x(2 h)=-\frac{\sqrt{h}}{\pi} h(h) .
\end{array}\right.
$$


Here, $\lambda>0, \alpha=\frac{1}{2}, p(t)=1$ and $f(t, x)=t^{2} x$, for $t \in[h, m h]_{h \mathbb{Z}}$ and $x \in \mathbb{R}$. By Remark 5, we find that the problem (8) is a private case of the problem (2) in $\mathbb{T}=h \mathbb{Z}$. Then (3) holds, and

$$
{ }_{h}^{h \mathbb{Z}} I_{t}^{1}|p(t)|+{ }_{h}^{h \mathbb{Z}} I_{t}^{\frac{1}{2}}|p(t)| \leq \frac{3 m}{2}(h-1) .
$$

If $3 m(h-1)<2 \lambda$, then $(4)$ holds, Thus, the conditions of Theorem 1 are satisfied, and we conclude that there is a function $x \in \mathcal{C}\left([h, m h]_{h \mathbb{Z}}, \mathbb{R}\right)$ the unique solution of (8).

\section{ACKNOWLEDGMENTS}

The authors would like to thank the anonymous reviewers for their valuable comments and suggestions to improve the quality of the manuscript.

\section{REFERENCES}

[1] A. Cabada, D. Vivero, Expression of the Lebesgue $\Delta$-integral on time scales as a usual Lebesgue integral; application to the calculus of $\Delta$-antiderivatives, Mathematical and Computer Modelling, 43 (2006), 194-207.

[2] A. Granas, J. Dugundji, Fixed point theory, Springer Monographs in Mathematics, Springer-Verlag, New York, 2003.

[3] A. B. Cherif, F. Z. Ladrani, Hardy's-sobolev's-type inequalities on time scale via alpha conformable fractional integral, Journal of Fractional Calculus and Applications, 2 (8) (2017), 156-165.

[4] A. B. Cherif, A. Hammoudi, F. Z. Ladrani, Density problems in $L_{\Delta}^{p}(\mathbb{T}, \mathbb{R})$ space, Electronic Journal of Mathematical Analysis and Applications, 1 (2013), 178-187.

[5] A. B. Cherif, F. Z. Ladrani, Density problems in sobolev's spaces on time scales, Kragujevac Journal of Mathematics, 45 (2021), 215-223.

[6] A. B. Cherif, F. Z. Ladrani, Asymptotic behavior of solution for a fractional RiemannLiouville differential equations on time scales, Malaya Journal of Matematik, 5 (3) (2017), 561-568.

[7] B. Bendouma, A. B. Cherif, A. Hammoud, Systems of first-order nabla dynamic equations on time scales, Malaya Journal of Matematik, 4 (6) (2018), 757-765.

[8] B. Bendouma, A. Hammoudi, Nonlinear Functional Boundary Value Problems for Conformable Fractional Dynamic Equations on Time Scales, Mediterranean Journal of Mathematics, 16, 25 (2019).

[9] D. Mozyrska, D. F. M. Torres, M. Wyrwas, Solutions of systems with the CaputoFabrizio fractional delta derivative on time scales, Nonlinear Analysis: Hybrid Systems, 32 (2019), 168-176.

[10] E. G. Bajlekova, Fractional evolution equations in Banach spaces, Eindhoven University of Technology, Eindhoven, 2001. 
[11] F. Z. Ladrani, A. B. Cherif, Oscillation Tests for Conformable Fractional Differential Equations with Damping, Punjab University Journal of Mathematics, 2 (52) (2020), $73-82$.

[12] G. M. Bahaa, D. F. M. Torres, Time-Fractional Optimal Control of Initial Value Problems on Time Scales, Nonlinear Analysis and Boundary Value Problems, 292 (2019), 229-242.

[13] I. Yaslan, Existence of positive solutions for second-order impulsive boundary value problems on time scales, Mediterranean Journal of Mathematics, 13 (2016), 1613-1624.

[14] I. Yaslan, O. Liceli, Three-point boundary value problems with delta RiemannLiouville fractional derivative on time scales, Fractional Differential Calculus, 6 (2016), 1-16.

[15] K. B. Oldham, A. J. Spanier, The Fractional Calculus: Theory and Applications of Differentiation and Integration to Arbitrary Order, New York, NY, USA: Academic Press, 1974.

[16] K. Mekhalfi, D. F. M. Torres, Generalized Fractional Operators on Time Scales with Application to Dynamic Equations, The European Physical Journal Special, 226 (2017), 3489-3499.

[17] M. Bohner, A. C. Peterson, Dynamic Equations on Time Scales, An Introduction with Applications, Birkäuser Boston, Inc., Boston, MA, 2001.

[18] M. Bohner, A. C. Peterson, Advances in Dynamic Equations on Time Scales, Birkäuser Boston, Inc., Boston, MA, 2003.

[19] M. R. Sidi Ammi, D. F. M. Torres, Existence and uniqueness results for a fractional Riemann-Liouville nonlocal thermistor problem on arbitrary time scales, Journal of King Saud University-Science, 30 (2018), 381-385.

[20] M. Belmekki, M. Benchohra, K. Ezzin, Existence results for some partial functional differential equations with state-dependent delay, Applied Mathematics Letters, 24 (2011), 1810-1816.

[21] N. Benkhettou, A. Hammoudi, D. F. M. Torres, Existence and uniqueness of solution for a fractional Riemann-Liouville initial value problem on time scales, Journal of King Saud University-Science, 28 (2016), 87-92.

[22] S. Hilger, Ein Maßkettenakalkül mit Anwendung auf Zentrumsmannigfaltigkeiten, Ph.D. Thesis, Universität Würzburg, 1988 (in German).

[23] S. Abbas, M. Benchohra, G. M. N'Gu'Gu'Gurrrkata, Topics in fractional differential equations, Developments in Mathematics, Springer, New York, 2012.

[24] S. Abbas, M, Benchohra, J. Graef, Integro-Differential Equations of Fractional Order, Differential Equations and Dynamical Systems, 20 (2012), 139-148.

[25] T. Abdeljawad, D. F. M. Torres, Symmetric duality for left and right RiemannLiouville and Caputo fractional differences, Arab Journal of Mathematical Sciences, 23 (2017), 157-172. 
[26] T. Gülse, E. Yilmaz, and H. Kemaloğlu, Conformable fractional Sturm-Liouville equation and some existence results on time scales, Turkish Journal of Mathematics, 42 (2018), 1348-1360.

[27] V. Kumar, M. Malik, Existence, Uniqueness and Stability of Nonlinear Implicit Fractional Dynamical Equation with Impulsive condition on Time Scales, Nonautonomous Dynamical Systems, 6 (1) (2019), 65-80.

[28] Y. Zhang, L. Ma, Solvability of Sturm-Liouville problems on time scales at resonance, Journal of Computational and Applied Mathematics, 233 (8) (2010), 1785-1797.

\author{
Amin Benaissa Cherif \\ Department of MAThematics \\ University of Science And Technology of Oran \\ "Mohamed-Boudiaf" (USTOMB) \\ ORAN \\ Algeria \\ E-mail address: amine.banche@gmail.com \\ amin.benaissacherif@univ-usto.dz
}

\title{
FAtima Zohra Ladrani
}

Department of Mathematics

Higher Training Teacher's School of Oran (ENSO)

ORAN

Algeria

E-mail address: f.z.ladrani@gmail.com 